\title{
Interview with Doron Zeilberger
}

Toufik Mansour

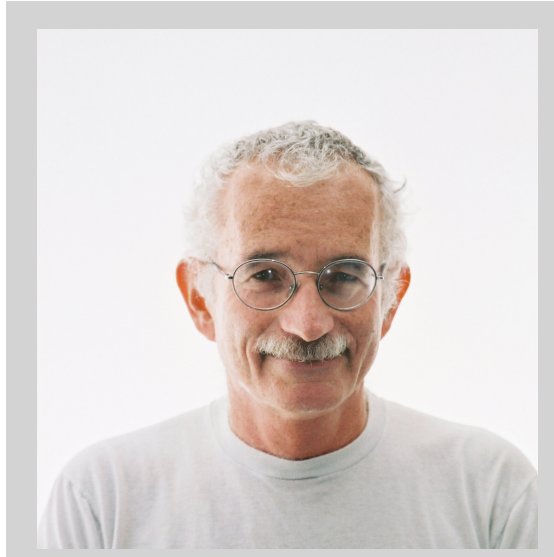

Doron Zeilberger received a B.Sc. in mathematics from the University of London in 1972, and a Ph.D. in mathematics from the Weizmann Institute of Science in 1976, under the supervision of Harry Dym. Professor Zeilberger has important contributions to the fields of hypergeometric summation and $q$-Series and was the first to prove the alternating sign matrix conjecture. He is considered a champion of using computers and algorithms to do mathematics quickly and efficiently, and his results have been used extensively in modern computer algebra software. Professor Zeilberger's distinctions include the Lester R. Ford Award in 1990, Leroy P. Steele Prize for Seminal Contributions to Research in 1998 for the development of WZ theory with Herbert Wilf, and the Euler Medal in 2004. In 2016 he received, together with Manuel Kauers and Christoph Koutschan, the David P. Robbins Prize of the American Mathematical Society. Professor Zeilberger was a member of the inaugural 2013 class of fellows of the American Mathematical Society.

Mansour: Professor Zeilberger, first of all we would like to thank you for accepting this interview. Would you tell us broadly what combinatorics is?

Zeilberger: Combinatorics is everything. All our worlds, the physical, mathematical, and even spiritual, are inherently finite and discrete, and so-called infinities, be their actual or potential, as well as the 'continuum', are 'optical illusions'.

Mansour: What do you think about the development of the relations between combinatorics and the rest of mathematics?

Zeilberger: Some of it is definitely good, but for sociological, psychological and political reasons, derived from the fact that (so far) mathematics is done (mostly) by humans, the obsession with 'relations to other parts of mathematics' has gone too far, and to a large part was driven from the need for respectability, and feeling part of the ruling- 'mainstream'.

Mansour: What have been some of the main goals of your research?

Zeilberger: Since, very soon, pure humangenerated mathematics will be done better and faster by computers, I dedicate my research to teaching computers to do mathematics, that in my case is mostly combinatorics and number theory. In fifty years, computers will not need us, but until then, it is fun to act as "coaches". Mansour: What were your early experiences with mathematics?

Zeilberger: See my interview with Ron Aharoni from $2015^{1}$.

Mansour: Were there specific problems that made you first interested in combinatorics?

Zeilberger: I started out in analysis, and tried to solve a problem, suggested by my advisor, Harry Dym, by doing a discrete analog. I never solved the original problem, but I fell in love with the discrete. To be honest I never liked the continuous, especially the way it was treated in the usual real analysis courses based on the 'theological' (and meaningless) notions

\footnotetext{
The authors: Released under the CC BY-ND license (International 4.0), Published: November 3, 2020

Toufik Mansour is a professor of mathematics at the University of Haifa, Israel. His email address is tmansour@univ.haifa.ac.il

${ }^{1}$ http://sites.math.rutgers.edu/ zeilberg/mamarim/mamarimhtml/netgar.html

${ }^{2}$ http://sites.math.rutgers.edu/ zeilberg/mamarim/mamarimhtml/real.html
} 
of 'real' numbers (what an oxymoron!) ${ }^{2}$.

Mansour: We would like to ask you about your formative years. What was the first reason you become interested in mathematics and specifically combinatorics? Did that happen under the influence of your family, or some other people?

Zeilberger: Again see ${ }^{3}$.

Mansour: What was the reason you chose the Weizmann Institute for your Ph.D. and your advisor, Harry Dym?

Zeilberger: I read a beautiful article that Harry wrote in Advances in Mathematics, on de-Branges spaces. Even though it was officially analysis, it had beautiful algebraic structure. At the end I winded up not working on it, but that was what lead me to Harry Dym.

Mansour: What was the problem you worked on in your thesis?

Zeilberger: As I said above, I tried to tackle a continuous problem by first doing a discrete analog hoping to 'take the limit' at the end, but then abandoned the original problem and worked on discrete analytic functions. See my Ph.D. thesis ${ }^{4}$.

Mansour: How was the mathematics at the Weizmann Institute at the time?

Zeilberger: Very stimulating. My teachers Harry Dym and Yakar Kannai were really inspiring.

Mansour: Would you say a little bit about your most influential results and why they have been influential?

Zeilberger: To use the cliche the whole is larger than the sum of its parts, I hope that my most significant contribution is in the realization, that the future of mathematics is in the direction of close collaboration with our sillicon friends, until they won't need us anymore.

Mansour: Would you tell us about your friend and collaborator Herbert Wilf and your joint works, specifically on the development of the Wilf-Zeilberger theory?

Zeilberger: He was a great mensch and a true giant. He was also ahead of his time, using computers and devising algorithms to handle combinatorial objects.
Mansour: What would guide you in your research? A general theoretical question or a specific problem?

Zeilberger: Whenever I see a problem that 'can be taught to a computer' I love it.

Mansour: When you are working on a problem, do you feel that something is true even before you have the proof?

Zeilberger: As I said many times, the notion of proof in mathematics is misguided and it should be called fully formal proofs. There are many statements that are obviously true, for example, Goldbach conjecture, the irrationality of Euler's constant, the Riemann Hypothesis, and $P \neq N P$, that are obviously true, but we human beings do not yet have a formal proof. Hopefully computers will help us soon.

Mansour: You criticize being too obsessed with rigorous mathematical proofs. Why?

Zeilberger: As I said above, and in more detail in the following essay ${ }^{5}$, the notion of "rigorous proof' has nothing to do with wanting to know the truth about the mathematical universe, it is just an (often fun, I admit) intellectual game, and also a competitive sport, and also a (fanatical) religious dogma.

Mansour: What three results do you consider the most influential in combinatorics during the last thirty years?

Zeilberger: These are too numerous to list, but I like those results that in hindsight turned out to have one-page proofs, that previously only had partial results with long and boring proofs.

- The Adam Marcus-Gabor Tardos's proof ${ }^{6}$ of the Stanley-Wilf conjecture ${ }^{7}$.

- The Ellenberg-Gijswijt's proof (based on a previous proof of Croot-Lev-Pach of an analogous result) about three-term arithmetical progressions ${ }^{8}$.

- The Hao Huang's ingenious proof of the sensitivity conjecture ${ }^{9}$.

These are true gems, and they also show the inherent triviality of human-generated mathematics. All these conjectures that great human minds could not do for many years, turned out to have one-page proofs.

\footnotetext{
${ }^{3}$ http://sites.math.rutgers.edu/ zeilberg/mamarim/mamarimhtml/netgar.html

${ }^{4}$ See http://sites.math.rutgers.edu/ zeilberg/DZthesis.pdf

${ }^{5}$ https://sites.math.rutgers.edu/ zeil berg/mamarim/mamarimhtml/hersh90.html

6 https://www.sciencedirect.com/science/article/pii/S0097316504000512?via\%3Dihub

7 https://sites.math.rutgers.edu/ ${ }^{\sim}$ zeilberg/mamarim/mamarimhtml/martar.html

8 https://sites.math.rutgers.edu/ ${ }^{\sim}$ zeilberg/mamarim/mamarimhtml/f $3 \mathrm{n} . \mathrm{html}$

${ }^{9}$ https://annals.math.princeton.edu/2019/190-3/p06
} 
Mansour: What are the top three open questions in your list?

Zeilberger: Let me answer a different question. The three steps in my research agenda are:

1. Make the mainstream obsolete.

2. Make the offbeat mainstream.

3. Go to 1.

Mansour: What kind of mathematics would you like to see in the next ten-to-twenty years as the continuation of your work?

Zeilberger: Computer-generated, or at least computer-assisted proof of

(i) the Riemann Hypothesis ${ }^{10}$

(ii) $P \neq N P$

(iii) the Collatz conjecture ${ }^{11}$

Mansour: What would you say about some of the major directions in combinatorics for the next two decades?

Zeilberger: I am not qualified to answer regarding normal human-generated combinatorics. My personal agenda is to teach your computer to do your math for you.

Unfortunately, if you want an academic job in a traditional math department, you need to do the same-old, currently mainstream research.

Mansour: You have "Doron Zeilberger's Collection of Quotes" on your web page. What is your favourite quote?

Zeilberger: It is hard, but how about " $M y$ occupation is an open question. I was once an assistant professor of mathematics. Since then, I have spent time living in the woods of Montana." -Theodore J. Kaczynski, New York Times Jan. 23, 1998, p. A18

Mansour: Would you tell us about your interests besides mathematics?

Zeilberger: Solving two-move chess problems. Since I was good at math I was expected to be a good chess player. The truth is that I was (and still am) terrible. Because of this inferiority complex, I started doing twomovers thirty years ago. Even in this endeavor I am still bad, but much better than I was thirty years ago. I soon will try to study threemovers. It also makes me appreciate my students who are not naturally good at math, but through hard work manage to do well.

Mansour: Before we close this interview with one of the foremost experts in combinatorics, we would like to ask some more specific mathematical questions. You have met several combinatorial objects throughout your career. Which one is your favourite?

Zeilberger: Permutations, and their cousins, alternating sign matrices.

Mansour: How do you define "Experimental Mathematics"?

Zeilberger: Experimental Mathematics used to be an oxymoron, but in the future it would be the opposite, a redundancy. All mathematics (at least the part that is worth doing) would be ipso facto, experimental.

Mansour: Would you tell us about your thought process for the proof of the alternating sign matrix conjecture? How did you become interested in this conjecture? How long did it take you to figure out a proof? Did you have a "eureka moment"?

Zeilberger: I heard it from Dave Robbins (1942-2003), one of my great heroes, at a combinatorics meeting at Oberwolfach, in 1982. It went through several iterations, where the referee, that turned out to be Dave Robbins himself, kept founding gaps. Luckily with the help of almost 90 checkers, and a detailed Maple package, all the gaps were filled. A few months later, Greg Kuperberg observed that it is an almost immediate corollary of a result in mathematical physics due to Izergin and Korepin, and the proof turned out to be 'almost' a triviality, but this did not detract from the original much longer proof that used the methodology of constant terms, and incidentally proved a much more general result (that $\mathrm{Gog}=$ Magog also for trapezoids, not only for triangles), for which the ad-hoc Kuperberg-Izergin-Korepin approach is not (as far as I know) applicable.

Mansour: The study of permutation patterns has seen great advances in the last thirty years. Any comments on the research in this direction?

Zeilberger: Yes indeed, it is a booming field, and you, Toufik, are a major player!

Mansour: Why is it very difficult to count some combinatorial objects? Do you think that someone will be able to compute the Stanley-Wilf limit corresponding to the pattern 1324 in the next decade?

\footnotetext{
${ }^{10}$ See http://www.claymath.org/millennium-problems/riemann-hypothesis

${ }^{11}$ See https://www.quantamagazine.org/why-mathematicians-still-cant-solve-the-collatz-conjecture-20200922/
} 
Zeilberger: Because life is hard. The few combinatorial objects that we can count exactly are the trivial ones (in the eyes of God). So even counting alternating sign matrices is trivial, compared to counting 1324-avoiding permutations. I doubt that the exact number of 1324-avoiding permutations with 10000 terms, the number of $10000 \times 10000$ Latin squares, or the number of self-avoiding walks of length 10000 will ever be known. Also the Stanley-Wilf limit would never be known to an accuracy of more than 20 decimal digits.

Mansour: You recently have an interesting paper titled as "A simple rederivation of Onsager's solution of the 2D Ising model using experimental mathematics." Do you expect some other interesting results in this direction?

Zeilberger: Yes. I hope (when I have time) that my coauthor, Manuel Kauers and I can generalize the method (with the help of our beloved computers) to the still open prob- lem with magnetic field, and the 3D version, thereby getting us to shake hands (once the pandemic is over) with the King of Sweden.

Mansour: Is there a specific problem you have been working on for many years? What progress have you made?

Zeilberger: The Riemann Hypothesis, the Collatz conjecture, $P \neq N P$. No luck, so far.

Mansour: Are you working on an interesting problem with your famous "co-author" Shalosh B. Ekhad these days?

Zeilberger: Yes, we try to count (with Manuel Kauers and his computer) Standard Young Tableaux with forbidden runs, that, contrary to the opinions of the editors of the journal Algebraic Combinatorics, are both deep and original.

Mansour: Professor Doron Zeilberger, I would like to thank you for this very interesting interview on behalf of the journal Enumerative Combinatorics and Applications. 